\title{
A theoretical investigation into the trapping of noble gases by clathrates on Titan
}

\author{
Caroline Thomas, Sylvain Picaud, \\ Olivier Mousis and Vincent Ballenegger \\ Université de Franche-Comté, Institut UTINAM, \\ CNRS/INSU, UMR 6213, 25030 Besançon Cedex, France
}

\begin{abstract}
In this paper, we use a statistical thermodynamic approach to quantify the efficiency with which clathrates on the surface of Titan trap noble gases. We consider different values of the $\mathrm{Ar}, \mathrm{Kr}, \mathrm{Xe}, \mathrm{CH}_{4}, \mathrm{C}_{2} \mathrm{H}_{6}$ and $\mathrm{N}_{2}$ abundances in the gas phase that may be representative of Titan's early atmosphere. We discuss the effect of the various parameters that are chosen to represent the interactions between the guest species and the ice cage in our calculations. We also discuss the results of varying the size of the clathrate cages. We show that the trapping efficiency of clathrates is high enough to significantly decrease the atmospheric concentrations of Xe and, to a lesser extent, of $\mathrm{Kr}$, irrespective of the initial gas phase composition, provided that these clathrates are abundant enough on the surface of Titan. In contrast, we find that $\mathrm{Ar}$ is poorly trapped in clathrates and, as a consequence, that the atmospheric abundance of argon should remain almost constant. We conclude that the mechanism of trapping noble gases via clathration can explain the deficiency in primordial Xe and Kr observed in Titan's atmosphere by Huygens, but that this mechanism is not sufficient to explain the deficiency in Ar.
\end{abstract}

Key words: Titan; Clathrates; Atmosphere; Noble gases; Cassini-Huygens

\section{Introduction}

Saturn's largest satellite, Titan, has a thick atmosphere, primarily consisting of nitrogen, with a few percent of methane (Niemann et al., 2005). An unexpected feature of this atmosphere is that no primordial noble gases, other than Ar, were detected by the Gas Chromatograph Mass Spectrometer (GCMS) aboard

Email address: caroline.thomas@univ-fcomte.fr (Caroline Thomas).

Preprint submitted to Planetary and Space Science 12 December 2018 
the Huygens probe during its descent on January 14, 2005. The detected Ar includes primordial ${ }^{36} \mathrm{Ar}$ (the main isotope) and the radiogenic isotope ${ }^{40} \mathrm{Ar}$, which is a decay product of ${ }^{40} \mathrm{~K}$ (Niemann et al., 2005). The other primordial noble gases ${ }^{38} \mathrm{Ar}$, Kr and Xe, were not detected by the GCMS instrument, yielding upper limits of $10^{-8}$ for their mole fractions in the gas phase. Furthermore, the value of the ${ }^{36} \mathrm{Ar} /{ }^{14} \mathrm{~N}$ ratio is about six orders of magnitude lower than the solar value, indicating that the amount of ${ }^{36} \mathrm{Ar}$ is surprisingly low within Titan's atmosphere (Niemann et al., 2005). These observations seem to be at odds with the idea that noble gases are widespread in the bodies of the solar system. Indeed, these elements have been measured in situ in the atmospheres of the Earth, Mars and Venus, as well as in meteorites (Owen et al., 1992). The abundances of Ar, Kr and Xe were also measured to be oversolar by the Galileo probe in the atmosphere of Jupiter (Owen et al., 1999).

In order to explain the observed deficiency of primordial noble gases in Titan's atmosphere, Osegovic \& Max (2005) proposed that the noble gases within the atmosphere could be trapped in clathrates located on the surface of Titan. The authors calculated the composition of clathrates on the surface of Titan using the program CSMHYD (developed by Sloan (1998)) and showed that such crystalline ice structures may act as a sink for Xe. The facts that the code used by Osegovic \& Max (2005) is not suitable below about $140 \mathrm{~K}$ for gas mixtures of interest, and that the authors did not explicitly calculate the trapping efficiencies of Ar and $\mathrm{Kr}$ in clathrates on the surface of Titan led Thomas et al. (2007) to reconsider their results. In particular, Thomas et al. (2007) performed more accurate calculations of the trapping of noble gases in clathrates using a statistical thermodynamic model based on experimental data and on the original work of van der Waals \& Platteeuw (1959). On this basis, Thomas et al. (2007) showed that Xe and Kr could have been progressively absorbed in clathrates located at the surface of Titan during its thermal history, in contrast with Ar, which is poorly trapped in these crystalline structures. They then concluded that their calculations are only partly consistent with the Huygens probe measurements, since the presence of clathrates on the surface of Titan cannot explain the primordial Ar deficiency in its atmosphere.

In this paper, we aim to extend the work of Thomas et al. (2007) by considering the clathration of a more plausible gas mixture representative of the composition of Titan's atmosphere, in which we include Ar, Kr and Xe all together. Indeed, Osegovic \& Max (2005) and Thomas et al. (2007) both considered atmospheric compositions containing only one noble gas at a time. As we shall see, the competition between the various species strongly affects the efficiency with which they are trapped in clathrates. In addition, because the incorporation conditions of guest species in clathrates depend on the structural characteristics of the crystalline network and on the intermolecular potentials, we examine the influence of the size of cages and of the interaction potential 
parameters on our calculations. The study of the influence of these parameters is motivated by two distinct facts. First, it is known that the size of the clathrate cages depends on the temperature (Shpakov et al., 1997; Belosludov, 2002; Takeya et al., 2005), and secondly, several different sets of Kihara parameters are available in the literature (Parrish \& Prausnitz, 1972; Diaz Peña et al., 1982; Jager, 2001). As a result, this study of sensitivity to parameters allows us to better quantify the accuracy of the results.

In Section 2, we describe the statistical model used to calculate the composition of the clathrates. This hybrid model is based on the work of van der Waals \& Platteeuw (1959), and on available experimental data. We also compare the dissociation pressure of clathrates obtained from our model with that obtained from the CSMHYD program (Sloan, 1998). CSMHYD uses a more sophisticated and rigorous approach, but is limited to carrying out calculations above about $140 \mathrm{~K}$ (Sloan, 1998). In Section 3, we investigate the sensitivity of our model to various parameters, and also examine the influence of these parameters on the predicted clathrate composition. In Section 4, the statistical approach developed in Section 2 is used to calculate the relative abundances of guests trapped in clathrates that may exist on the surface of Titan. Several hypotheses for the abundance of noble gases in the atmosphere of Titan are tested. Section 5 is devoted to the summary and discussion of our results.

\section{Theoretical background}

To calculate the relative abundance of guest species incorporated in a clathrate from a coexisting gas of specified composition at given temperature and pressure, we follow the formalism developed by Lunine \& Stevenson (1985), which is based on the statistical mechanics approach of van der Waals \& Platteeuw (1959). Such an approach relies on four key assumptions: the host molecules contribution to the free energy is independent of the clathrate occupancy (this assumption implies in particular that the guest species do not distort the cages), the cages are singly occupied, there are no interactions between guest species in neighboring cages, and classical statistics is valid, i.e., quantum effects are negligible (Sloan, 1998).

In this formalism, the occupancy fraction of a guest species $G$ for a given type $t(t=$ small or large) of cage, and for a given type of clathrate structure (I or II) can be written as:

$$
y_{G, t}=\frac{C_{G, t} P_{G}}{1+\sum_{J} C_{J, t} P_{J}},
$$

where $C_{G, t}$ is the Langmuir constant of guest species $G$ in the cage of type 
$t$, and $P_{G}$ is the partial pressure of guest species $G$. Note that this assumes that the sample behaves as an ideal gas. The partial pressure is given by $P_{G}=x_{G} \times P$, with $x_{G}$ the molar fraction of guest species $G$ in the initial gas phase, and $P$ the total pressure. The sum, $\sum_{J}$, in the denominator runs over all species $J$ which are present in the initial gas phase.

The Langmuir constants indicate the strength of the interaction between each guest species and each type of cage. This interaction can be accurately described, to a first approximation, on the basis of the spherically-averaged Kihara potential $w_{G}(r)$ between the guest species $G$ and the water molecules forming the surrounding cage (McKoy \& Sinanoğlu, 1963), written as:

$$
\begin{aligned}
w_{G}(r) & =2 z \epsilon_{G} \frac{\sigma_{G}^{12}}{R_{c}^{11} r}\left(\delta_{G}^{(10)}(r)+\frac{a_{G}}{R_{c}} \delta_{G}^{(11)}(r)\right) \\
& -\frac{\sigma_{G}^{6}}{R_{c}^{5} r}\left(\delta_{G}^{(4)}(r)+\frac{a_{G}}{R_{c}} \delta_{G}^{(5)}(r)\right)
\end{aligned}
$$

where $R_{c}$ represents the radius of the cavity assumed to be spherical. $z$ is the coordination number of the cell and $r$ the distance from the guest molecule to the cavity center. The parameters $R_{c}$ and $z$ depend on the structure of the clathrate and on the type of the cage (small or large), and are given in Table 1. The functions $\delta_{G}^{(N)}(r)$ are defined as :

$$
\delta_{G}^{(N)}(r)=\frac{1}{N}\left[\left(1-\frac{r}{R_{c}}-\frac{a_{G}}{R_{c}}\right)^{-N}-\left(1+\frac{r}{R_{c}}-\frac{a_{G}}{R_{c}}\right)^{-N}\right] .
$$

where $a_{G}, \sigma_{G}$ and $\epsilon_{G}$ are the Kihara parameters for the interactions between guest species and water. The choice of the Kihara parameters for the guest species considered in the present study is discussed in the next section. The parameters chosen for our calculations are given in Table 2.

The Langmuir constants are then determined by integrating the Kihara potential within the cage as

$$
C_{G, t}=\frac{4 \pi}{k_{B} T} \int_{0}^{R_{c}} \exp \left(-\frac{w_{G}(r)}{k_{B} T}\right) r^{2} d r
$$

where $T$ represents the temperature and $k_{B}$ the Boltzmann constant.

Finally, the relative abundance $f_{G}$ of a guest species $G$ in a clathrate (of structure I or II) is defined as the ratio of the average number of guest molecules of species $G$ in the clathrate over the average total number of enclathrated 
molecules :

$$
f_{G}=\frac{b_{s} y_{G, s}+b_{\ell} y_{G, \ell}}{b_{s} \sum_{J} y_{J, s}+b_{\ell} \sum_{J} y_{J, \ell}},
$$

where the sums in the denominator run over all species present in the system, and $b_{s}$ and $b_{\ell}$ are the number of small and large cages per unit cell, respectively. Note that the relative abundances of guest species incorporated in a clathrate can differ strongly from the composition of the coexisting gas phase because each molecular species has a different affinity with the clathrate.

The calculations are performed at temperature and pressure conditions at which the multiple guest clathrates are formed. The corresponding temperature and pressure values $\left(T=T_{\text {mix }}^{\text {diss }}\right.$ and $\left.P=P_{\text {mix }}^{\text {diss }}\right)$ can be read from the dissociation curve of the multiple guest clathrates.

In the present study, the dissociation pressure is determined from available experimental data and from a combination rule due to Lipenkov \& Istomin (2001). Thus, the dissociation pressure $P_{\text {mix }}^{\text {diss }}$ of a multiple guest clathrate is calculated from the dissociation pressure $P_{G}^{\text {diss }}$ of a pure clathrate of guest species $G$ as

$$
P_{\operatorname{mix}}^{\text {diss }}=\left(\sum_{G} \frac{x_{G}}{P_{G}^{\text {diss }}}\right)^{-1}
$$

where $x_{G}$ is the molar fraction of species $G$ in the gas phase.

The dissociation pressure $P_{G}^{\text {diss }}$ is derived from laboratory measurements and follows an Arrhenius law (Miller, 1961):

$$
\log \left(P_{G}^{\text {diss }}\right)=A+\frac{B}{T}
$$

where $P_{G}^{\text {diss }}$ and $T$ are expressed in $\mathrm{Pa}$ and $\mathrm{K}$, respectively. The constants $A$ and $B$ used in the present study have been fitted to the experimental data given by Lunine \& Stevenson (1985) and by Sloan (1998) and are listed in Thomas et al. (2007).

The present approach differs from that proposed by Sloan (1998) in the CSMHYD program, in which the dissociation pressure of the multiple guest clathrate is calculated in an iterative way by requiring that the chemical potential in the clathrate phase is equal to that in the gas phase. The determination of this equilibrium requires knowledge of the thermodynamics of an empty hydrate, such as the chemical potential, enthalpy and volume difference between ice (chosen as a reference state) and the empty hydrate. The experimental 
data available at standard conditions $(T=273.15 \mathrm{~K}, P=1 \mathrm{~atm})$ allow the CSMHYD program to calculate chemical potentials, and hence dissociation curves, as long as the temperature and pressure is not too far from the reference point. This method fails to converge at temperatures below $140 \mathrm{~K}$ for the clathrates considered in this study. Our approach avoids this problem, because it uses experimentally determined dissociation curves, which are valid down to low temperatures.

As an illustration, Fig. 1 shows a comparison between the dissociation curve obtained from our approach (full line) and that calculated using the CSMHYD program (crosses) for a multiple guest clathrate corresponding to an initial gas phase composition of $4.9 \% \mathrm{CH}_{4}, 0.1 \% \mathrm{C}_{2} \mathrm{H}_{6}$ and $95 \% \mathrm{~N}_{2}$. For this comparison, both calculations have been performed with the same set of parameters (Kihara parameters and cage geometries given by Sloan, 1998). Both methods give very similar results in the range where the CSMHYD program converges.

\section{Sensitivity to parameters}

The present calculations of the relative abundances of guest species incorporated in a clathrate depend on the structural characteristics of this clathrate (size of the cages for example) and also on the parameters of the Kihara potential. It is thus useful to assess the influence of these structural characteristics and potential parameters on the calculations, in order to better quantify the accuracy of the results.

\subsection{Discussion of the Kihara parameters}

Papadimitriou et al. (2006) have recently illustrated the sensitivity of clathrate equilibrium calculations to Kihara parameters values, in the case of methane and propane clathrates. Indeed, by perturbing in the range $\pm(1 \%-10 \%)$ the $\sigma$ and $\epsilon$ Kihara parameters originally given in Sloan (1998), they have demonstrated that these parameters have a significant effect on the values of the Langmuir constants and on the dissociation curves. It appears thus of fundamental importance to assess the accuracy of the Kihara parameters which are used in the studies of clathrates, for example by comparing the theoretical results with available experimental data. However, because in the present paper we are interested in the calculations of the relative abundances of noble gases

in clathrates on Titan which are not experimentally available, our choice of Kihara parameters has been based on the literature, only.

For the guest species considered here, i.e., $\mathrm{CH}_{4}, \mathrm{C}_{2} \mathrm{H}_{6}, \mathrm{~N}_{2}$, Ar, $\mathrm{Kr}$, and Xe, 
as far as we know, there are only two full sets of Kihara parameters in the literature (Parrish \& Prausnitz, 1972; Diaz Peña et al., 1982) which have been used in the context of clathrate studies. These parameters are unfortunately very different (see Table 2). The parameters given by Parrish \& Prausnitz (1972) have been obtained by comparing calculated chemical potentials based on the structural data of the clathrates cages given by von Stackelberg \& Müller (1954) with experimental results based on clathrate dissociation pressure data (Parrish \& Prausnitz, 1972). The parameters given by Diaz Peña et al. (1982) have been fitted on experimentally measured interaction virial coefficients for binary mixtures. They have been recently used by Iro et al. (2003) to quantify the trapping by clathrates of gases contained in volatiles observed in comets. The corresponding calculations were performed by using the clathrate cage parameters given by Sparks et al. (1999). These two sets of Kihara parameters (Parrish \& Prausnitz, 1972; Diaz Peña et al., 1982) can be partly compared to those recently given for $\mathrm{CH}_{4}, \mathrm{C}_{2} \mathrm{H}_{6}, \mathrm{~N}_{2}$, and $\mathrm{Xe}$, only, in the PhD work of M. Jager (2001).

Table 2 shows that the $\sigma$ and $\epsilon$ parameters used by Jager (2001) are quite close to those given by Parrish \& Prausnitz (1972) and, as a consequence, the relative abundances $f_{G}$ (with $G=\mathrm{CH}_{4}, \mathrm{C}_{2} \mathrm{H}_{6}, \mathrm{~N}_{2}$, or Xe) we have calculated with these two sets of parameters are of the same order of magnitude, and behave similarly with temperature. By contrast, the relative abundances calculated with the parameters given by Diaz Peña et al. (1982) are very different from those calculated with the two other sets of Kihara parameters, as expected from the conclusions of Papadimitriou et al. (2006).

Because the potential and structural parameters given by Parrish \& Prausnitz (1972) (Table 1) have been self-consistently determined on experimentally measured clathrates properties, and also because they give results similar to those obtained when using Jager's parameters (Jager, 2001) for a reduced set of species, we choose here the Parrish \& Prausnitz's parameters for the rest of our study.

\subsection{Influence of the size of the cages}

In the present paper, we have chosen the potential and structural parameters given by Parrish \& Prausnitz (1972) (Table 1). However, it has been shown that the size of the cages can vary as a function of the temperature (thermal expansion or contraction) and also of the size of the guest species (Shpakov et al., 1997; Takeya et al., 2005; Belosludov et al., 2002; Hester et al., 2007). Indeed, the structural parameter $R_{c}$ increases with temperature and with the size of the guest species, whereas it decreases when small guest species are

encaged. For example, the lattice constant of the methane hydrate is increased 
by $0.3 \%$ between 83 and $173 \mathrm{~K}$ (Takeya et al., 2005).

In order to quantify the influence of variations of the size of the cages on the relative abundances calculated for the multiple guest clathrate considered in the present study, we have modified by $\pm(1 \%-5 \%)$ the $R_{c}$ values given in Table 1 . These variations are compatible with typical thermal expansion or contraction in the temperature range 90-270 K (Shpakov et al., 1997; Takeya et al., 2005; Belosludov et al., 2002; Hester et al., 2007).

Figure 2 shows the evolution with temperature of $f_{\mathrm{Ar}}, f_{\mathrm{Kr}}$ and $f_{\mathrm{Xe}}$ (calculated from Eq. 5) in a multiple guest clathrate, for both structures I and II, and for variations of the size of the cages equal to $\pm 1 \%$ and $\pm 5 \%$. The calculations have been performed for an initial gas phase containing $\mathrm{CH}_{4}, \mathrm{C}_{2} \mathrm{H}_{6}, \mathrm{~N}_{2}, \mathrm{Ar}$, $\mathrm{Kr}$ and $\mathrm{Xe}$. The gas phase abundance of $\mathrm{CH}_{4}(4.92 \%)$ has been taken from Niemann et al. (2005), whereas the values for $\mathrm{C}_{2} \mathrm{H}_{6}(0.1 \%), \operatorname{Ar}(0.1 \%), \mathrm{Kr}$ $(0.1 \%)$ and $\mathrm{Xe}(0.1 \%)$ are based on our previous study (Thomas et al., 2007). The relative abundance of $\mathrm{N}_{2}(94.68 \%)$ has been determined accordingly.

Our results show that a small variation $( \pm 1 \%)$ of the size of the cages has only a very small effect on the trapping of noble gases in the corresponding multiple guest clathrate, irrespective of the temperature (Fig. 2). In particular, the behavior (i.e., increase or decrease) with temperature is not affected by small variations of the size of the cages. A similar small effect is also observed for an expansion of the cage by $5 \%$. By contrast, a large contraction of the cages $\left(R_{c}\right.$ decreased by $5 \%$ ) leads to strong modifications of the relative abundances $f_{G}$ $(G=\mathrm{Ar}, \mathrm{Kr}, \mathrm{Xe})$ which can vary by several orders of magnitude.

The evolution of the relative abundances of all guests in the clathrates is also given in Fig. 3 as a function of the sizes of the cages, for given pressure and temperature. The corresponding calculations have been performed at a pressure $P=1.5$ bar (i.e., the present atmospheric pressure at the surface of Titan), and at the corresponding temperature given by the dissociation curve, i.e., $T_{\mathrm{mix}}^{\mathrm{diss}}=176 \mathrm{~K}$ (see below). Figure 3 clearly shows that, for the given $P$ and $T$ conditions, the contractions of the cages have a larger effect than the expansions on the relative abundances calculated in clathrates and that these effects are also strongly dependent on the interaction parameters between the guest species and the cages. As a consequence, the relative abundances in clathrates of the noble gases considered in the present paper appear to be much more dependent on the size of the cages than those calculated for $\mathrm{CH}_{4}$, $\mathrm{C}_{2} \mathrm{H}_{6}$ and $\mathrm{N}_{2}$. This feature can be related to the values of the $\epsilon$ parameters of the Kihara potential (see Table 2) which are larger for the noble gases than for the other species. Moreover, the relative abundances of the smallest species (i.e., the noble gases which have the smallest values of the $\sigma$ Kihara parameters) are increased when decreasing the sizes of the cages. 
To summarize, the results given in Figs. 2 and 3 show that thermal variations of the cages need to be taken into account if these variations are greater than a few percents. Unfortunately, these variations with temperature are often not known, except for a small number of specific systems, such as the methane clathrate between 83 and $173 \mathrm{~K}$ (Takeya et al., 2005) for which the variations of the cages have been shown to be very small. In a first approximation, such thermal variations will thus be neglected in our calculations of the $f_{G}$ evolution on Titan, as a function of the temperature.

\section{Trapping of noble gases by clathrates on Titan}

The statistical approach outlined in Section 2 is used to calculate the relative abundances of $\mathrm{CH}_{4}, \mathrm{C}_{2} \mathrm{H}_{6}, \mathrm{~N}_{2}, \mathrm{Ar}, \mathrm{Kr}$ and $\mathrm{Xe}$ in a multiple guest clathrate (structures I and II), as a function of the temperature. As discussed above, the interactions between the guests and the surrounding cages have been calculated by using the Kihara potential with the parameters given by Parrish \& Prausnitz (1972), and by disregarding the possible influence of the thermal variations of the cages.

The initial gas phase abundance of $\mathrm{CH}_{4}(4.92 \%)$ is taken from Niemann et al. (2005), whereas three different sets of initial abundances are considered for $\mathrm{N}_{2}, \mathrm{C}_{2} \mathrm{H}_{6}, \mathrm{Ar}, \mathrm{Kr}$, and Xe in the atmosphere of Titan. The first set of values (hereafter case 1) is derived from the atmospheric composition considered by Osegovic \& Max (2005) and was also used in our previous study (Thomas et al., 2007). The second set of values is calculated under the assumption that each ratio of noble gas to methane gas in the atmosphere of Titan corresponds to the solar abundance (Lodders 2003) with all carbon postulated to be in the form of methane (hereafter case 2). The third set of values is calculated under the assumption that each noble gas to methane gas phase ratio derives from the value calculated by Alibert \& Mousis (2007) for planetesimals produced in the feeding zone of Saturn and ultimately accreted by the forming Titan (hereafter case 3). In each case, the relative abundance of $\mathrm{N}_{2}$ (approximately $95 \%$ ) has been determined such as $x_{\mathrm{CH}_{4}}+x_{\mathrm{Ar}}+x_{\mathrm{Kr}}+x_{\mathrm{Xe}}+x_{\mathrm{N}_{2}}+x_{\mathrm{C}_{2} \mathrm{H}_{6}}=1$. The initial gas phase abundances for the three cases are given in Table 3.

Figure 4 shows that the dissociation curves calculated for the multiple guest clathrates that form in the three considered atmospheres exhibit a similar behavior, although for a given temperature, the dissociation pressure can vary by two orders of magnitude from one case to another one (especially at low temperatures). However, for a pressure corresponding to the present pressure at the surface of Titan (i.e., $P=1.5$ bar), the dissociation temperatures given by Fig. 4 for the three cases are within a $20 \mathrm{~K}$ range, with corresponding

values $T_{\text {mix }}^{\text {diss }}=176,167$, and $185 \mathrm{~K}$, for cases 1,2 and 3 , respectively. These 
results indicate that the influence of the initial abundances in the gas phase is quite weak on the stability of the corresponding multiple guest clathrate.

Then, we have calculated the variations with temperature of the relative abundances $f_{G}$ in the multiple guest clathrate considered in the present study, in each case.

Figure 5 shows the corresponding results calculated for a multiple guest clathrate of structure I or II. For case 1, this figure shows that the relative abundances of $\mathrm{Ar}, \mathrm{Kr}, \mathrm{CH}_{4}$ and $\mathrm{N}_{2}$ decrease when the formation temperature of the clathrate decreases, in contrast with the relative abundances of $\mathrm{Xe}$ and $\mathrm{C}_{2} \mathrm{H}_{6}$ which slightly increase when the temperature decreases. This indicates that the efficiency of the trapping by multiple guest clathrates decreases with temperature for Ar and Kr, whereas it slightly increases for Xe. This result differs from that obtained in our previous study (Thomas et al., 2007) in which the trapping of both $\mathrm{Xe}$ and $\mathrm{Kr}$ was found to increase when the temperature decreases. However, in this previous study, we performed the calculations by assuming the presence of only one noble gas in the initial gas phase, the two others being excluded. The difference obtained in the present study for a gas phase containing the three noble gases, indicates that there is a strong competition between the trapping of $\mathrm{Xe}, \mathrm{Kr}$, and Ar, when considering that they can be trapped all together. Similar conclusions are obtained when considering the two other cases, although the trapping of Xe is found to increase much more than in case 1 when the temperature decreases. Also, in cases 2 and 3, the trapping of $\mathrm{Kr}$ appears almost constant in the whole range of temperatures considered in the present study. Note that the absolute values of the relative abundances are very different for the three cases due to the different compositions of the initial gas phase.

As a consequence, it is much more useful to compare the efficiency of the trapping mechanism in each case by calculating the abundance ratios for the three considered noble gases Ar, Kr and Xe. Such a ratio is defined as in our previous paper (Thomas et al., 2007), i.e., as the ratio between the relative abundance $f_{G}$ of a given noble gas in the multiple guest clathrate and its initial gas phase abundance $x_{G}$ (see Table 3 ). The ratios calculated for Xe, $\mathrm{Kr}$, and $\mathrm{Ar}$ in the three cases considered here are given in Table 4. These ratios have been calculated at the particular point on the dissociation curves (Fig. 4) corresponding to the present atmospheric pressure at the ground level of Titan (i.e., $P=1.5$ bar and $T=T_{\text {mix }}^{\text {diss }}$ ).

Table 4 shows that for this particular point of the dissociation curve, the relative abundances of $\mathrm{Xe}$ and $\mathrm{Kr}$ trapped in multiple guest clathrates are much higher than in the initial gas phase, irrespective of the initial gas phase composition. By contrast, the relative abundance of Ar is similar in gas phase and in the multiple guest clathrate. These results indicate that the efficiency 
of the trapping by clathrate may be high enough to significantly decrease the atmospheric concentrations of $\mathrm{Xe}$ and, to a lesser extent, of $\mathrm{Kr}$, irrespective of the initial gas phase composition, provided that clathrates are abundant enough at the surface of Titan. On the contrary, with an abundance ratio close to 1 , Ar is poorly trapped in clathrates and the Ar atmospheric abundance consequently should remain almost constant.

\section{Summary and discussion}

We have extended the work of Thomas et al. (2007) by considering the clathration of a gas mixture presumably representing the composition of Titan's atmosphere, where Ar, $\mathrm{Kr}$ and Xe are included all together. In this context, we have developed a hybrid statistical model derived from the works of van der Waals \& Platteeuw (1959) and Lipenkov \& Istomin (2001), and using available experimental data to constrain the clathrates composition. Because it has been shown that clathrates equilibrium calculations are very sensitive to the guest species - cage interaction potential, we have compared different sets of potential parameters existing in the literature. Our calculations were performed using the parameters calculated by Parrish \& Prausnitz (1972), because these parameters form a consistent set for our application to clathrates on Titan. We have also assessed the influence of the thermal variations of the size of the cages to better quantify the accuracy of the composition prediction. We show that these variations need to be taken into account if they are greater than a few percents. We have then considered several initial gas phase compositions, including different sets of noble gases abundances, that may be representative of Titan's early atmosphere. We finally show that the trapping efficiency of clathrates is high enough to significantly decrease the atmospheric concentrations of $\mathrm{Xe}$ and, in a lesser extent, of $\mathrm{Kr}$, irrespective of the initial gas phase composition, provided that these clathrates are abundant enough at the surface of Titan. On the contrary, with an abundance ratio close to 1 , Ar is poorly trapped in clathrates and its atmospheric abundance should remain consequently almost constant. Despite the fact that we consider simultaneously three noble gases in the gas phase composition, in contrast with Thomas et al. (2007), we obtain the same conclusions: the noble gases trapping mechanism via clathration can explain the deficiency in primordial Xe and Kr observed by Huygens in Titan's atmosphere, but not that in Ar.

We note that, even if the Visible and Infrared Mapping Spectrometer (VIMS) onboard Cassini was able to see the surface unimpeded, the bulk composition of Titan's crust is still unknown. Hence, the presence of clathrates on the surface of Titan is difficult to quantify.

Thomas et al. (2007) calculated that the total sink of Xe or Kr in clathrates 
would represent a layer at the surface of Titan whose equivalent thickness would not exceed $\sim 50 \mathrm{~cm}$. The sink of these noble gases in clathrates requires the presence of available crystalline water ice on the surface or in the near subsurface of Titan. If an open porosity exists within the top few hundreds meters in the icy mantle of Titan, by analogy with the terrestrial icy polar caps, the amount of available water ice in contact with the atmosphere of Titan would thus increase and help the formation of clathrates inside the pores. Moreover, in presence of methane clathrate on the surface of Titan, diffusive exchange of noble gases with methane might occur in the cavities, thus favoring their trapping in clathrates. One must also note that the efficiency of the noble gases trapping by clathrates on Titan can be limited by the very slow (and poorly known) kinetics at these low temperatures and the availability of water ice to clathration. It is then difficult to estimate the timescale needed to remove the proposed quantities of noble gases from the atmosphere of Titan.

To explain the deficiency in Ar in a way consistent with the present results, we can invoke the Titan's formation scenario proposed by Alibert \& Mousis (2007) and Mousis et al. (2007). According to this scenario, the lack of CO in the atmosphere of Titan can be explained if Titan was formed from planetesimals that have been partially vaporized in the Saturn's subnebula. The vaporization temperature in the Saturn's subnebula $(\sim 50 \mathrm{~K})$ needed to explain the loss of $\mathrm{CO}$ in planetesimals ultimately accreted by Titan is also high enough to imply the sublimation of Ar and, in a lower extent, that of $\mathrm{Kr}$ (see e.g. Fig. 9 of Alibert \& Mousis, 2007). Indeed, Kr can also be partially trapped in methane clathrates formed in the solar nebula (Mousis et al., 2007). On the other hand, Xe still remains trapped in planetesimals because its incorporation occurs in conditions close to those required for the methane clathration in the nebula (Alibert \& Mousis, 2007; Mousis et al., 2007).

It is important to mention that the composition of Titan's today atmosphere is almost certainly different from that in past. In particular, the nitrogen isotopes in Titan's atmosphere suggest significant mass loss over time. Moreover, we do not know the outgassing history of methane. In particular, if the methane outgassing is recent (Tobie et al., 2006), the lack of infrared opacity prior to that era must result in freezing out of nitrogen. It is likely that the composition, pressure and temperatures in Titan's atmosphere have differed significantly in the past, which will affect the composition and formation efficiency of clathrates on the surface.

Finally, we note that there remains the possibility that the noble gas abundances are telling a story that entirely differs from the scenario we propose, in which neither molecular nitrogen nor noble gases were initially accreted in clathrates (Atreya et al., 2006; 2007). In this context, Titan would have formed from solids produced at such high temperatures that they would have accreted nitrogen essentially as ammonia hydrate. Planetesimals formed in such con- 
ditions would be directly impoverished in noble gases since their trapping in clathrates require lower temperature and pressure conditions (Atreya et al., 2006; 2007).

\section{Acknowledgements}

O.M. acknowledges the support of the French "Centre National d'Etudes Spatiales" (CNES). Support from the PID program "Origines des Planètes et de la Vie" of the CNRS is also gratefully acknowledged. We also acknowledge Dr. Jonathan Horner for a careful reading of the manuscript.

\section{References}

Alibert, Y., Mousis, O., 2007. Formation of Titan in Saturn's subnebula: constraints from Huygens probe measurements. Astronomy and Astrophysics 465, 1051-1060

Atreya, S. K., Matson, D. L., Castillo-Rogez, J., Johnson, T. V., Adams, E. Y., Lunine, J. I., 2007. Photochemical Origin of Nitrogen on Titan and Enceladus. AGU Fall Meeting Abstracts 3.

Atreya, S. K., Adams, E. Y., Niemann, H. B., Demick-Montelara, J. E., Owen, T. C., Fulchignoni, M., Ferri, F., Wilson, E. H., 2006. Titan's methane cycle. Planetary and Space Science 54, 1177-1187.

Belosludov, V. R., Inerbaev, T. M., Subbotin, O. S., Belosludov, R. V., Kudoh, J., Kawazoe, Y., 2003. Thermal expansion and lattice distortion of clathrate hydrates of cubic structures I and II. Journal of Supramolecular Chemistry 2 (4-5), 453-458.

Diaz Peña, M., Pando, C., Renuncio, J. A. R., 1982. Combination rules for intermolecular potential parameters. I. Rules based on approximations for the long-range dispersion energy. Journal of Chemical Physics 76, 325-332.

Hester, K.C., Huo, Z., Ballard, A.L., Koh, C.A., Miller, K.T., and Sloan, E.D., 2007. Thermal expansivity for sI and sII clathrate hydrates. Journal of Physical Chemistry B 111, 8830-8835.

Iro, N., Gautier, D., Hersant, F., Bockelée-Morvan, D., Lunine, J. I., 2003. An interpretation of the nitrogen deficiency in comets. Icarus 161, 511-532.

Jager, M., 2001. High pressure studies of hydrate phase inhibition using Raman spectroscopy. Ph.D. Thesis.

Lipenkov, V. Ya., Istomin, V. A., 2001. On the stability of air clathrate-hydrate crystals in subglacial Lake Vostok, Antartica. Materialy Glyatsiol. Issled. 91, 129-133.

Lodders, K., 2003. Solar System Abundances and Condensation Temperatures of the Elements. Astrophysical Journal 591, 1220-1247. 
Lunine, J. I., Stevenson, D. J., 1985. Thermodynamics of clathrate hydrate at low and high pressures with application to the outer solar system. Astrophysical Journal Supplement Series 58, 493-531.

McKoy, V., Sinanoğlu, O., 1963. Theory of dissociation pressures of some gas hydrates. Journal of Chemical Physics 38 (12), 2946-2956.

Miller, S. L., 1961. The occurence of gas hydrates in the solar system. Proceedings of the National Academy of Science 47 (11),1798-1808.

Mousis, O., Alibert, Y., Benz, W., 2006. Saturn's internal structure and carbon enrichment. Astronomy and Astrophysics 449, 411-415.

Mousis, O., Lunine, J. I., Thomas, C., Alibert, Y., 2007. Constraints On The Origin Of Titan From Huygens Probe Measurements. AAS/Division for Planetary Sciences Meeting Abstracts 39, \#44.09.

Niemann, H. B., and 17 colleagues, 2005. The abundances of constituents of Titan's atmosphere from the GCMS instrument on the Huygens probe. Nature 438, 779-784.

Osegovic, J. P., Max, M. D., 2005. Compound clathrate hydrate on Titan's surface. Journal of Geophysical Research (Planets) 110, 8004.

Owen, T., Bar-Nun, A., Kleinfeld, I. 1992. Possible cometary origin of heavy noble gases in the atmospheres of Venus, earth, and Mars. Nature 358, 43-46.

Owen, T., Mahaffy, P., Niemann, H. B., Atreya, S., Donahue, T., Bar-Nun, A., de Pater, I. 1999. A low-temperature origin for the planetesimals that formed Jupiter. Nature 402, 269-270.

Papadimitriou, N. I., Tsimpanogiannis, I. N., Yiotis, A. G., Steriotis, T. A., Stubos, A. K., 2007. On the use of the Kihara potential for hydrate equilibrium calculations. In: Kuhs, W. (Ed.), Physics and Chemistry of Ice. Proceedings of the 11th International Conference on the Physics and Chemistry of Ice 311, 475-482.

Parrish, W. R., Prausnitz, J. M., 1972. Dissociation pressures of gas hydrates formed by gas mixtures. Industrial and Engineering Chemistry: Process Design and Development, 11 (1), 26-35. Erratum : Parrish, W. R., Prausnitz, J. M., 1972. Industrial and Engineering Chemistry: Process Design and Development 11 (3), 462.

Shpakov, V. P., Tse, J. S., Tulk, C. A., Kvamme, B., Belosludov, V. R., 1998. Elastic moduli calculation and instability in structure I methane clathrate hydrate. Chemical Physics Letters 282 (2), 107-114.

Sloan, E. D., Jr., 1998. Clathrate hydrates of natural gases. Dekker, M. (Ed.), New York.

Sparks, K. A., Tester, J. W., Cao, Z., Trout, B. L., 1999. Configurational properties of water clathrates: Monte carlo and multidimensional integration versus the lennard-jones and devonshire approximation. Journal of Physical Chemistry B 103 (30), 6300-6308.

Takeya, S., Kida, M., Minami, H., Sakagami, H., Hachikubo, A., Takahashi, N., et al., 2006. Structure and thermal expansion of natural gas clathrate hydrates. Chemical Engineering Science 61 (8), 2670-2674.

Thomas, C., Mousis, O., Ballenegger, V., Picaud, S., 2007. Clathrate hydrates 
as a sink of noble gases in Titan's atmosphere. Astronomy and Astrophysics 474, L17-L20.

Tobie, G., Lunine, J. I., Sotin, C., 2006. Episodic outgassing as the origin of atmospheric methane on Titan. Nature 440, 61-64.

van der Waals, J. H., Platteeuw, J. C., 1959. Clathrate solutions. In: Advances in Chemical Physics, Vol. 2, Interscience, New York, 1-57.

von Stackelberg, M., Müller, H. R., 1954. Feste Gashydrate II. Struktur und Raumchemie. Elektrochemie 58, 25-39. 
Table 1

Parameters for the cavities. $R_{c}$ is the radius of the cavity (values taken from Parrish $\&$ Prausnitz, 1972). $b$ represents the number of small $\left(b_{s}\right)$ or large $\left(b_{\ell}\right)$ cages per unit cell for a given structure of clathrate (I or II), $z$ is the coordination number in a cavity.

\begin{tabular}{lcccc}
\hline \hline Clathrate structure & \multicolumn{2}{c}{ I } & \multicolumn{2}{c}{ II } \\
\hline Cavity type & small & large & small & large \\
$R_{c}(\AA)$ & 3.975 & 4.300 & 3.910 & 4.730 \\
$b$ & 2 & 6 & 16 & 8 \\
$z$ & 20 & 24 & 20 & 28 \\
\hline
\end{tabular}


Table 2

Three different sets of parameters for the Kihara potential. $\sigma$ is the Lennard-Jones diameter, $\epsilon$ is the depth of the potential well, and $a$ is the radius of the impenetrable core. These parameters come from the papers of (a) Parrish \& Prausnitz (1972), (b) Diaz Peña et al. (1982) and (c) Jager (2001).

\begin{tabular}{lllll}
\hline \hline Ref & Molecule & $\sigma(\AA)$ & $\epsilon / k_{B}(\mathrm{~K})$ & $a(\AA)$ \\
\hline (a) & $\mathrm{CH}_{4}$ & 3.2398 & 153.17 & 0.300 \\
& $\mathrm{C}_{2} \mathrm{H}_{6}$ & 3.2941 & 174.97 & 0.400 \\
& $\mathrm{~N}_{2}$ & 3.2199 & 127.95 & 0.350 \\
& $\mathrm{Xe}$ & 3.1906 & 201.34 & 0.280 \\
& $\mathrm{Ar}$ & 2.9434 & 170.50 & 0.184 \\
& $\mathrm{Kr}$ & 2.9739 & 198.34 & 0.230 \\
\hline (b) & $\mathrm{CH}$ & 3.019 & 205.66 & 0.313 \\
& $\mathrm{C}_{2} \mathrm{H}_{6}$ & 3.038 & 399.07 & 0.485 \\
& $\mathrm{~N}_{2}$ & 2.728 & 145.45 & 0.385 \\
& $\mathrm{Xe}$ & 3.268 & 302.49 & 0.307 \\
& $\mathrm{Ar}$ & 2.829 & 155.30 & 0.226 \\
& $\mathrm{Kr}$ & 3.094 & 212.70 & 0.224 \\
\hline (c) & $\mathrm{CH} H_{4}$ & 3.1514 & 154.88 & 0.3834 \\
& $\mathrm{C}_{2} \mathrm{H}_{6}$ & 3.2422 & 189.08 & 0.5651 \\
& $\mathrm{~N}_{2}$ & 3.0224 & 127.67 & 0.3526 \\
& $\mathrm{Xe}$ & 3.3215 & 192.95 & 0.2357 \\
& $\mathrm{Ar}$ & - & - & - \\
& $\mathrm{Kr}$ & - & - & - \\
\hline & & & &
\end{tabular}


Table 3

Three initial gas phase abundances corresponding to the systems considered by Osegovic \& Max (2005) (case 1), the solar nebula (case 2), and Saturnian planetesimals (case 3).

\begin{tabular}{lccc}
\hline \hline \multirow{2}{*}{ Molecule } & \multicolumn{3}{c}{ Molar fractions (\%) } \\
& case 1 & case 2 & case 3 \\
\hline $\mathrm{Ar}$ & 0.1 & $7.1264 \times 10^{-2}$ & 2.10506 \\
$\mathrm{Kr}$ & 0.1 & $3.44 \times 10^{-5}$ & $1.38 \times 10^{-3}$ \\
$\mathrm{Xe}$ & 0.1 & $3.8 \times 10^{-6}$ & $1.6 \times 10^{-4}$ \\
$\mathrm{CH}_{4}$ & 4.92 & 4.92 & 4.92 \\
$\mathrm{~N}_{2}$ & 94.68 & 95 & 92 \\
$\mathrm{C}_{2} \mathrm{H}_{6}$ & 0.1 & $8.6978 \times 10^{-3}$ & 0.9734 \\
\hline
\end{tabular}


Table 4

Abundance ratios of noble gas in clathrates to noble gas in the initial gas phase for $\mathrm{Ar}, \mathrm{Kr}$ and Xe. These ratios are calculated at $P=1.5 \mathrm{bar}$, and at the corresponding temperature on the dissociation curves (see Fig.4). This temperature is equal to 176 $\mathrm{K}$ for case $1,167 \mathrm{~K}$ for case 2 , and $185 \mathrm{~K}$ for case 3 .

\begin{tabular}{llcc}
\hline \hline Case & $\begin{array}{l}\text { Initial molar fraction } \\
\text { in gas }\end{array}$ & $\begin{array}{c}\text { abundance ratio } \\
\text { structure I }\end{array}$ & $\begin{array}{c}\text { abundance ratio } \\
\text { structure II }\end{array}$ \\
\hline & Ar gas & 0.4 & 1.5 \\
1 & $0.1 \times 10^{-2}$ & 0.8 & 5 \\
2 & $7.1264 \times 10^{-4}$ & 0.7 & 4.6 \\
3 & $2.10506 \times 10^{-2}$ & & \\
& Kr gas & 7.6 & 35.3 \\
1 & $0.1 \times 10^{-2}$ & 18.3 & 143.7 \\
2 & $3.44 \times 10^{-7}$ & 11.6 & 89.5 \\
3 & $1.38 \times 10^{-5}$ & & 473 \\
\hline & $\mathrm{Xe} \mathrm{gas}$ & 308 & 2356 \\
1 & $0.1 \times 10^{-2}$ & 863.4 & 948.7 \\
\hline & $3.8 \times 10^{-8}$ & 269.2 & \\
\hline
\end{tabular}




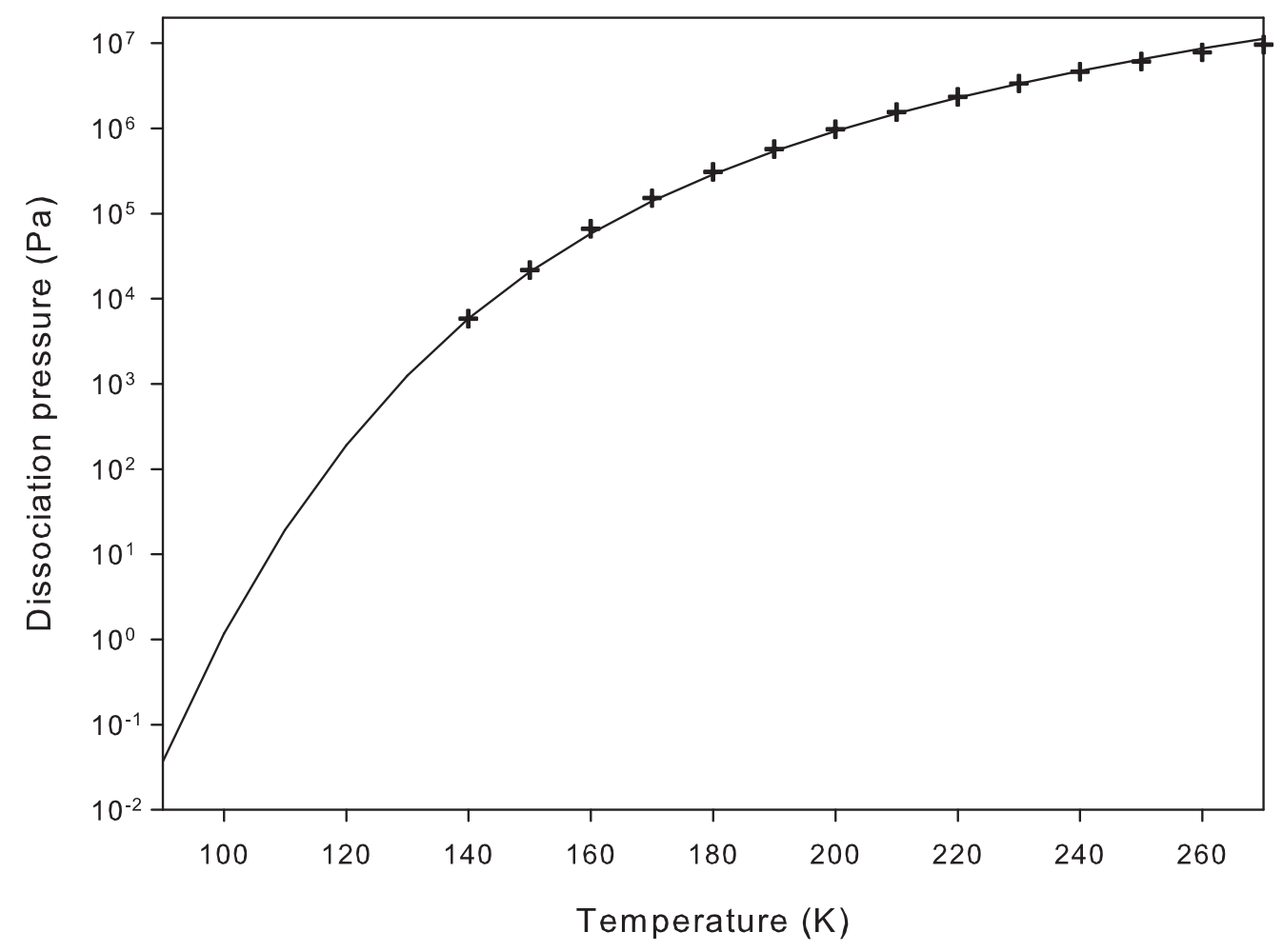

Fig. 1. Dissociation curves pressure as a function of temperature for a multiple guest clathrate corresponding to an initial gas phase composition of $4.9 \%$ of $\mathrm{CH}_{4}$, $0.1 \%$ of $\mathrm{C}_{2} \mathrm{H}_{6}$ and $95 \%$ of $\mathrm{N}_{2}$. The calculations have been performed using either the approach discussed in the present paper (full line) or the CSMHYD program proposed by Sloan (1998) (crosses). 


\section{Structure I}
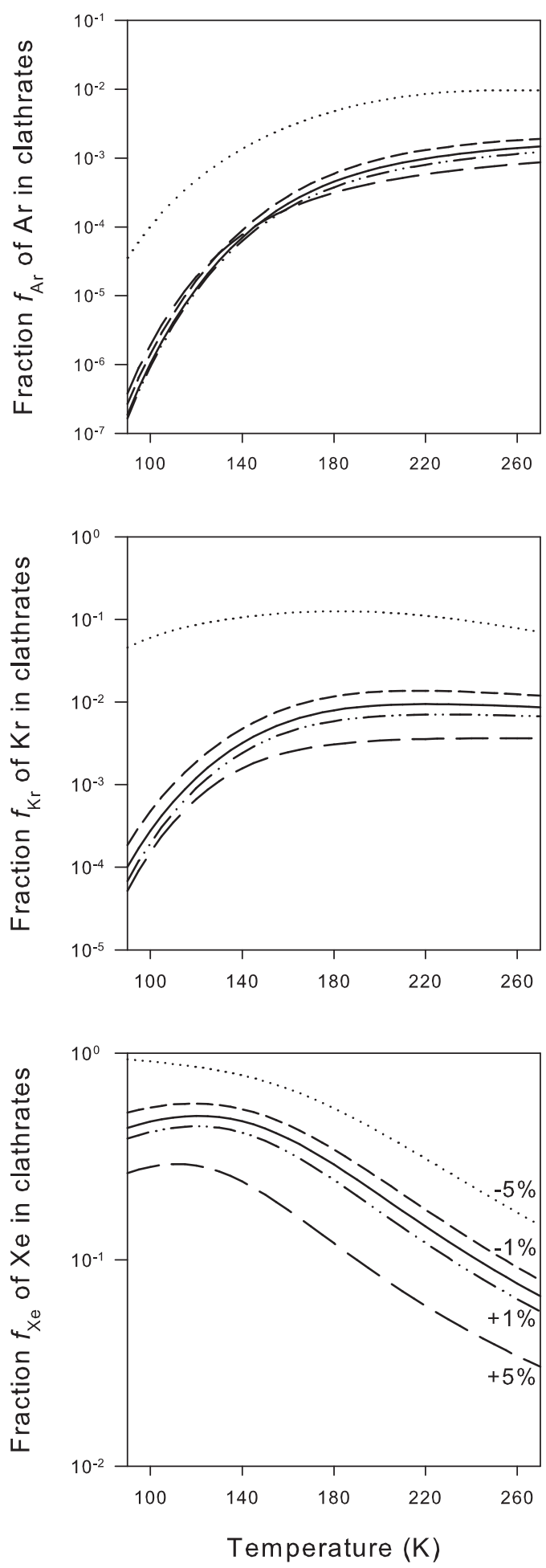

Structure II
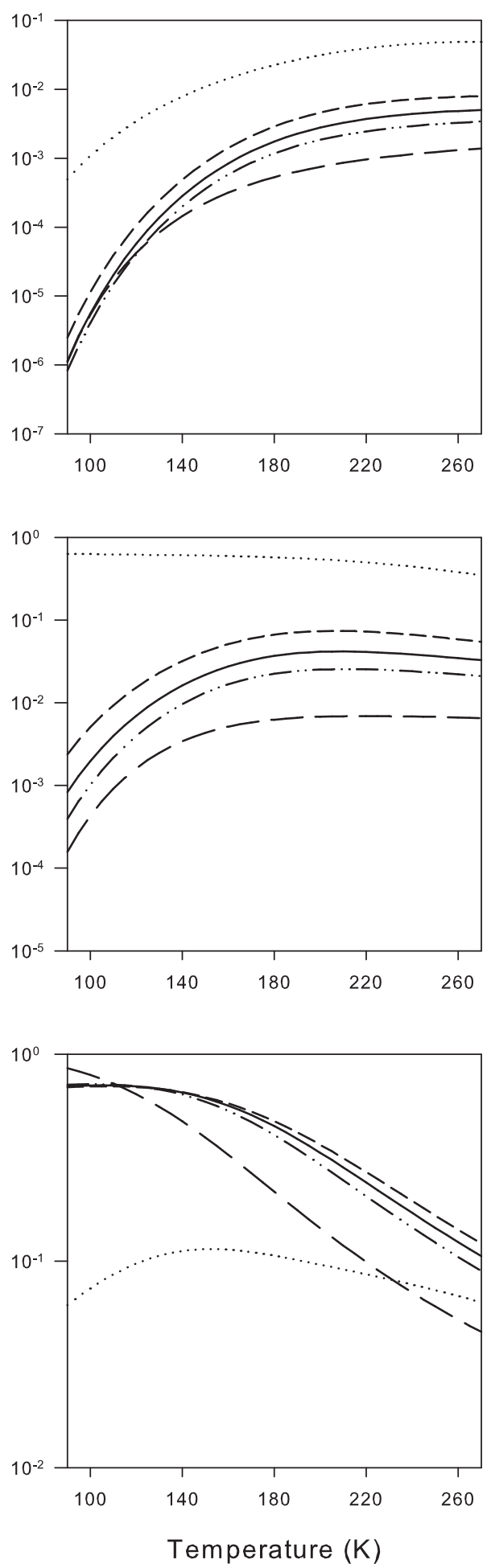

Fig. 2. Relative abundances of Ar, Kr, and Xe in clathrates as a function of temperature for structures I and II. The solid lines represent the results obtained with the parameters of the cages given in Table 1. The dash-dot-dotted and long dashed lines correspond to calculations performed with size of the cages increased by respectively $1 \%$ and $5 \%$. The medium dashed and dotted lines are results obtained with size of the cages decreased respectiyely by $1 \%$ and $5 \%$. 

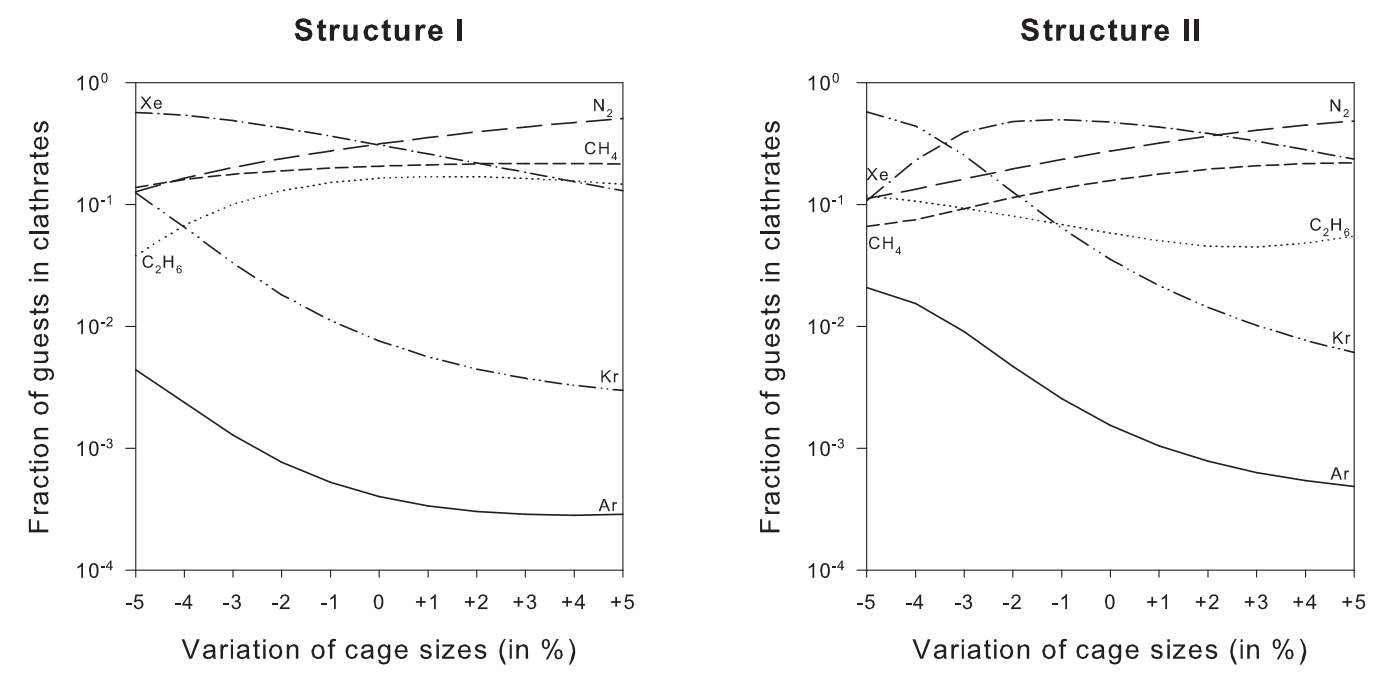

Fig. 3. Relative abundances of guests in clathrates as a function of the cage sizes. These results have been calculated at $P=1.5$ bar, corresponding to a dissociation temperature $\mathrm{T}=176 \mathrm{~K}$. 


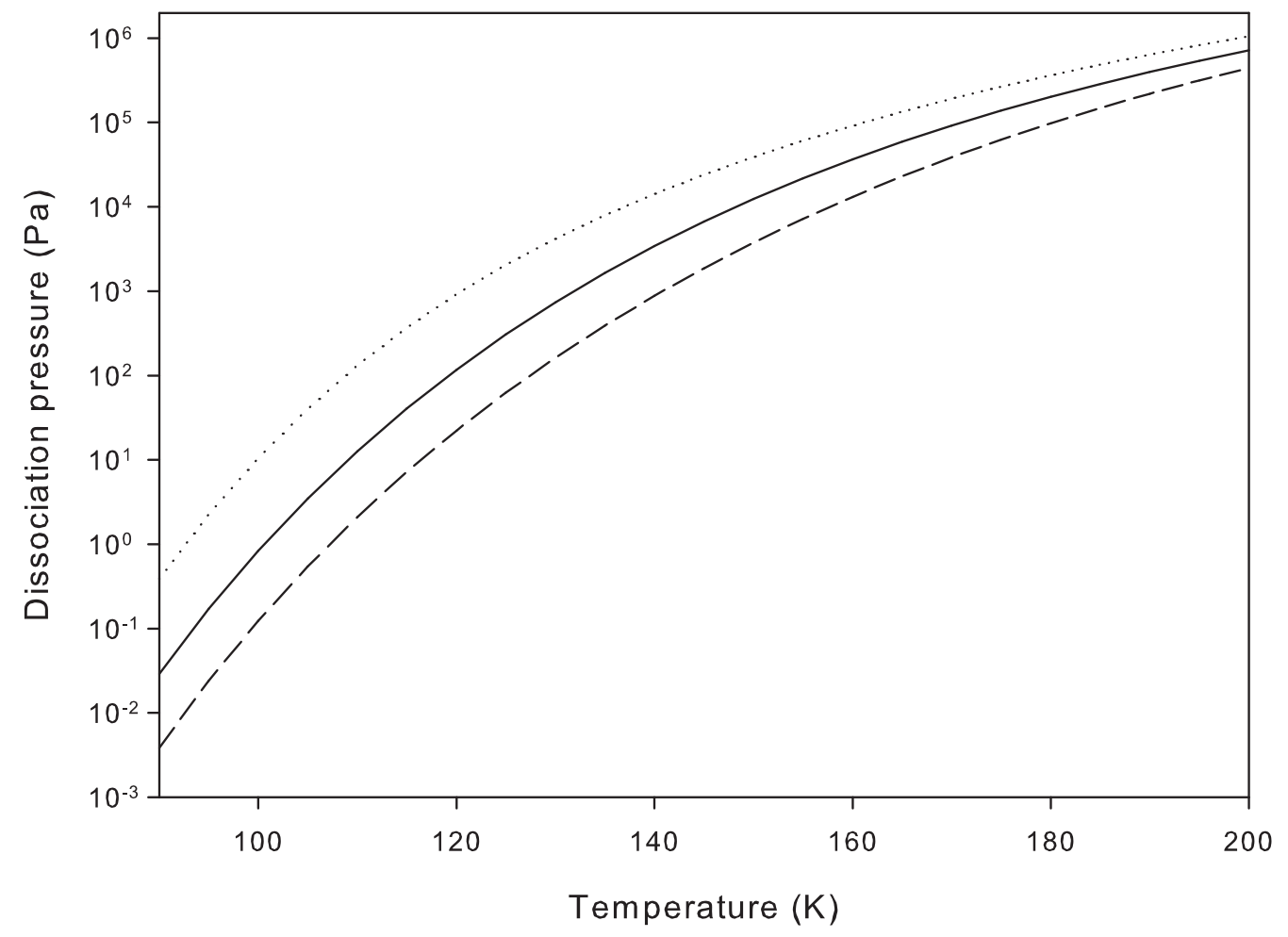

Fig. 4. Dissociation pressures of multiple guest clathrates, as a function of temperature for the three cases considered in the present study (see text) : case 1 (solid line), case 2 (dotted line), and case 3 (dashed line). 
Structure I
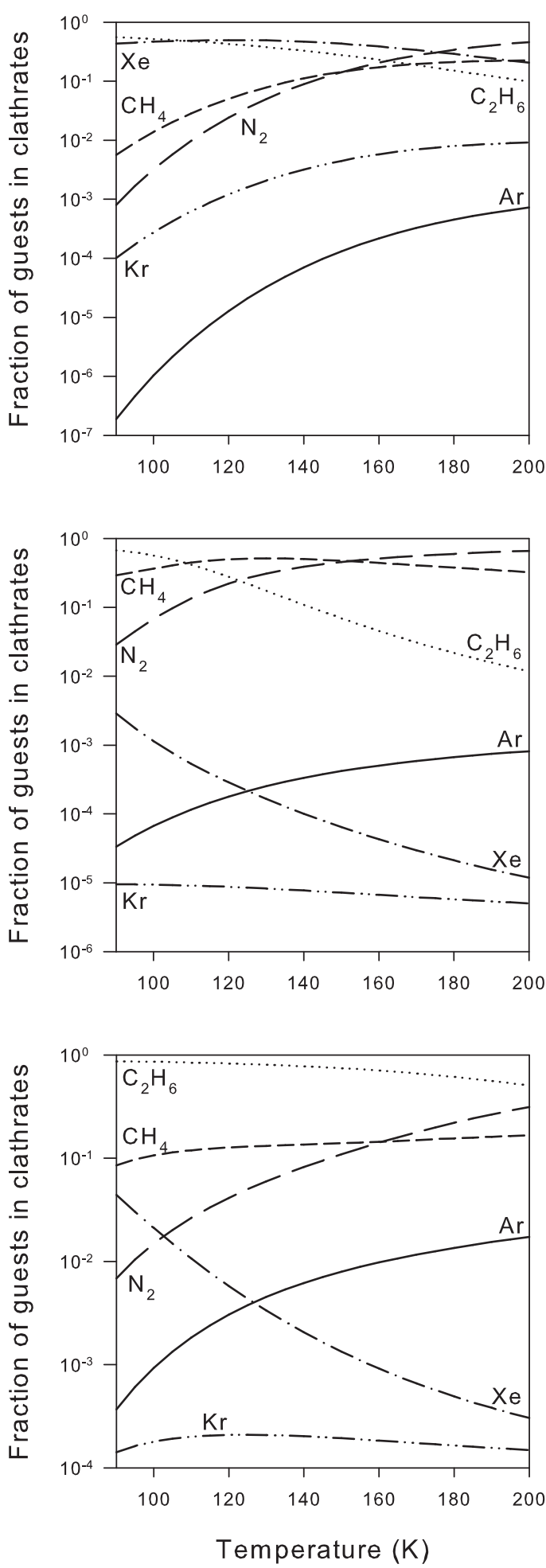

Structure II
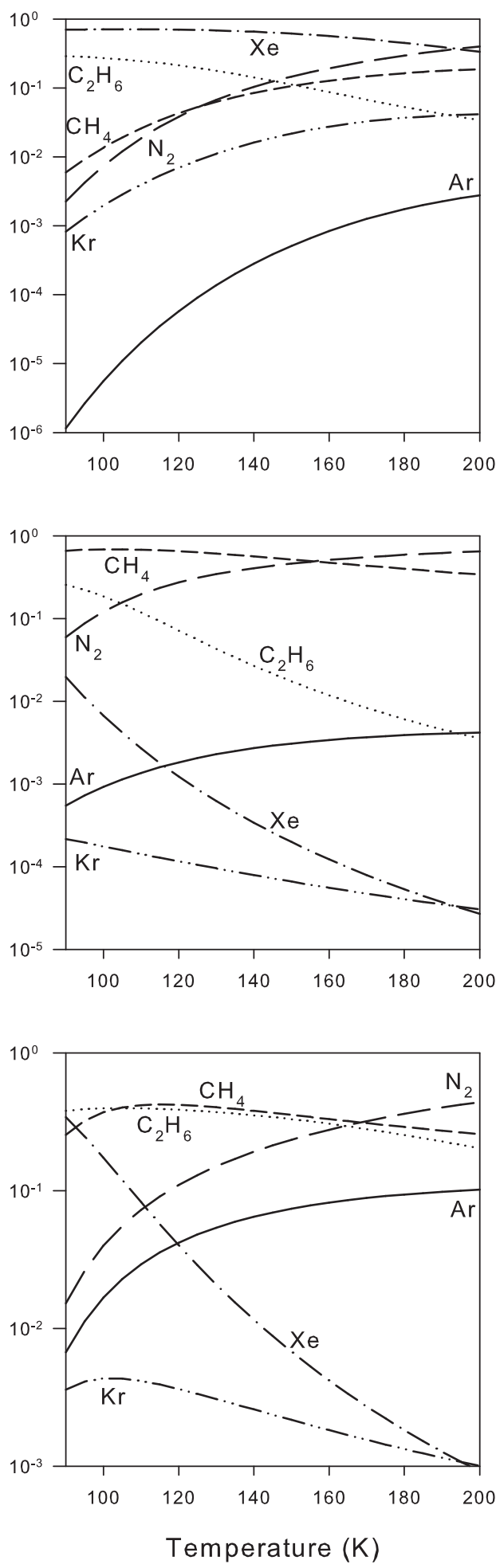

Fig. 5. Fractions of guests in clathrates, as a function of temperature for structures I and II; (top) case 1, (middle) case 2, (bottom) case 3. 